\title{
Microstructural aspects in steel fiber reinforced acrylic emulsion polymer modified concrete
}

\author{
Dayang Hazimmah ${ }^{1 *}$, Afizah $\mathrm{Ayob}^{2}$, Lau Sie Yee ${ }^{1}$, and Wong Chee Cung ${ }^{1}$ \\ ${ }^{1}$ School of Engineering and Technology, University College of Technology Sarawak, 868 Persiaran \\ Brooke, 96000 Sibu, Sarawak \\ ${ }^{2}$ Programme of Civil Engineering, School of Environmental Engineering, Universiti Malaysia Perlis, \\ 02600 Arau, Perlis, Malaysia
}

\begin{abstract}
Scanning electron microscope observations of polymer-free and polymer-modified cements have shown that the polymer particles are partitioned between the inside of hydrates and the surface of anhydrous cement grains. For optimum dosage of acrylic emulsion polymer with $2.5 \%$, the C-S-H gel in this structure is finer and more acicular. Some polymer adheres or deposit on the surface of the C-S-H gel. The presence of acrylic emulsion polymer confines the ionic diffusion so that the $\mathrm{Ca}(\mathrm{OH})_{2}$ crystallized locally to form fine crystals. The void in the structures seems to be smaller but no polymer films appears to be bridging the walls of pores although many polymer bonds or C-S-H spread into the pore spaces. In addition to porosity reduction, acrylic emulsion polymer modified the hydration products in the steel fiber -matrix ITZ. The hydration product $\mathrm{C}-\mathrm{S}-\mathrm{H}$ appeared as a needle like shape. The needleshaped C-S-H increases and gradually formed the gel, with needles growing into the pore space. The phenomenon is more obvious as curing age increased.
\end{abstract}

\section{Introduction}

Polymer modified cementitious mixtures (PMC) have been called by various names such as polymer Portland cement concrete (PPCC) and latex modified concrete (LMC) [1]. Polymer modified cementitious mixtures includes polymer modified cementitious slurry, mortar and concrete. The improvement from adding polymer modifier to concrete includes increased bond strength, freezing and thawing resistance, abrasion resistance, flexural and tensile strength and reduced permeability and elastic modulus [1]. A reduced elastic modulus will result in reducing the stresses developed due to differential shrinkage and thermal strain that would reduce the tendency of the material to crack. PMC can also have increased resistance to penetration by water and dissolve salts and reduced need for

\footnotetext{
*Corresponding author: siti.hazimmah@ucts.edu.my
} 
sustained moist curing. The improvements are measurably reduced when PMC is tested in the wet state [2].

Fiber reinforced concrete (FRC) is an alternative building material as it can strengthen the crack resistance and the toughness of the concrete effectively, and it is capable of reinforcing the impermeability and effectively eliminating the stress concentration of concrete as well [3]. Whereas, with the progression of the research and application, the existing issues of FRC are equally striking. Inside FRC, especially in the interface transition zone (ITZ) between fiber and cement substrate, there tend to be a large quantity if harmful pores, giving rise to the weak bonding among cement hydrates, fibers and aggregates [14]. Herein, the role of the fiber is deteriorated in enhancement $[4,5]$.

Fortunately, with the polymeric materials science and in-depth insights of the correlations and causations between material structures and properties progressing, large quantities of excellent properties possessed by polymer, such as waterproof, filling, flocculation and thickening effects were discovered. It is to be noted that polymer has been already implemented or gradually applied to concrete field currently [6,7]. With the purpose of making concrete capable of meeting the requirements of structural applications, the inclusion of polymer in concrete has become one of the focused research topics in engineering circles [8].

Different amounts of polymer addition are required to gain various improved properties of mortar or concrete. As far as mechanical properties or impermeability is concerned, a polymer addition of $3 \%-20 \%$ is usually needed. With less than $3 \%$ of polymer addition, the mechanical properties of impermeability hardly change. Impermeability can only be improved when the polymer forms a semi-continuous film in the matrix of hardened cement hydrates. Hence, a minimum polymer addition is required to ensure film formation, which is usually larger than $3 \%$ of $\mathrm{p} / \mathrm{c}$ ratio [9]. After the film formation of the polymer and the hardening of the mortar, a gradient polymer distribution is generated from the surface to the interior region of the mortar. A significantly condensed surface is obtained in situ, which provides a radically effective protective layer. These research objectives are to study the effect of acrylic emulsion polymer and steel fiber addition on mechanical properties and thermal properties of steel fiber reinforced acrylic emulsion polymer modified concrete (SFRPMC).

\section{Experimental}

The process of mixing and casting of the composites were carried out following [10]. The amount of acrylic emulsion polymer was kept ready in suitable container. The quantity of water was then measured with a measuring cylinder and kept in suitable container. All polymers were mixed with half of water that measured into another container and put into the pan mixer. The mixing time was let to be 1 minute to 2 minutes.

Then the amount of cement, silica fume and aggregates was kept ready in suitable container. Sand was fed into the pan mixer, a part of the coarse aggregates, cement, silica fume and the materials were mixed for 2 minutes. The other half of water with some of the remainder of the coarse aggregates was fed so as to break up any modules of mortar. After all ingredients were added, the mixing time was set to be 3 to 5 minutes till homogeneous obtained. The calculated steel fibers were measured and kept in suitable container. The steel fibers with $60 \mathrm{~mm}$ length sized hooked end were added in percentage by weight (of total wet solid). The fibers were added in small increments by sprinkling them onto the surface of the mix until all the fibers absorbed into the matrix. This technique was performed to prevent 'balling' or 'interlocking' of the fibers. 
From each experiments of steel fiber reinforced polymer modified cement concrete, there were consisted of three cylinders $(150 \times 300 \mathrm{~mm})$, three cubes $(100 \mathrm{~mm})$ and three beams $(100 \times 100 \times 500 \mathrm{~mm})$. After conducting slump test, the concrete mix is placed inside the prepared molds. Then, all specimens were compacted by means of a vibrating table. After that, the specimens were demolded after 24 hours and subjected to water curing until the testing date.

With the same percentages of mix proportions in different curing aging of 1 hour, 24 hours and 168 hours, a few small specimens of steel fiber reinforced acrylic emulsion polymer modified concrete with 1 mix of basic concrete were also tested for microstructural performance by using Scanning Electron Microscopy (SEM) with Energy Dispersive X-ray Analysis (EDX). In this experiment, for each formulation 6 specimens were fabricated for each test. The micrographs of the specimens of were recorded using SEM (Hitachi with S$3400 \mathrm{~N}$ ) and ZEISS SUPRA 55VP connected to an analysis system with EDX.

\section{Results and discussions}

Morphology examination by using SEM has been conducted on composite system that had been produced. The microstructures of tested specimens are shown in Fig. 1 to 3. It can be seen that due to the presence of polymer film in all the specimens, the bond among steel fibers, aggregates and cement is greatly improved. Obviously, the porosity and pore size distribution are influenced by the corporation of polymer. The overall porosity increases with the increasing dosage of polymer [11].

The SEM image of polymer modification cement shows a quite different morphology. It can be seen the fracture surface has more fibrillar outgrowths, or more recticular networks. Fig. 1 shows well defined hydrates with some C-S-H gel and the $\mathrm{Ca}(\mathrm{OH})_{2}$ crystals in the layer form, some C-S-H gel in the fiber form. It seems that they spread into the pore space, but do not completely fill the space and leave large voids.

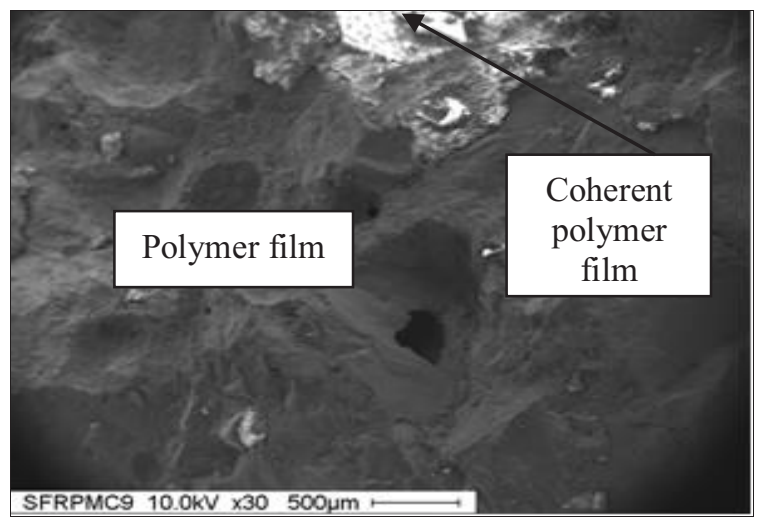

Fig. 1. Microstructure on mix 26 (1.0\% acrylic emulsion polymer, $8.0 \%$ silica fume, $1.5 \%$ steel fiber and $0.50 \mathrm{w} / \mathrm{c}$ ratio).

Fig. 2 shows the gross structure of polymer-cement paste that can be seen and the C-S$\mathrm{H}$ gel in this structure is finer and more acicular. It seems that some polymer adheres or deposit on the surfaces of the C-S-H gel. Needles of C-S-H appear to spread out from a cluster of hydrates and interweave together forming a crossing network. An additional observation of these figures is the morphology and dimension of $\mathrm{Ca}(\mathrm{OH})_{2}$ crystals is shown in Fig. 2. A group of fine $\mathrm{Ca}(\mathrm{OH})_{2}$ crystal are observed in the limited areas. The presence 
of polymer confines the ionic diffusion so that the $\mathrm{Ca}(\mathrm{OH})_{2}$ crystallized locally to form fine crystals. The void in the structures seems to be smaller, but no polymer films appears to be bridging the walls of the pores although many polymer bonds or C-S-H gel spread into the pore space. Due to the presence of polymer films in specimens, the bond among steel fibers, aggregates and cement is greatly improved [11] and leads to the highest strength for $\operatorname{mix} 43$.

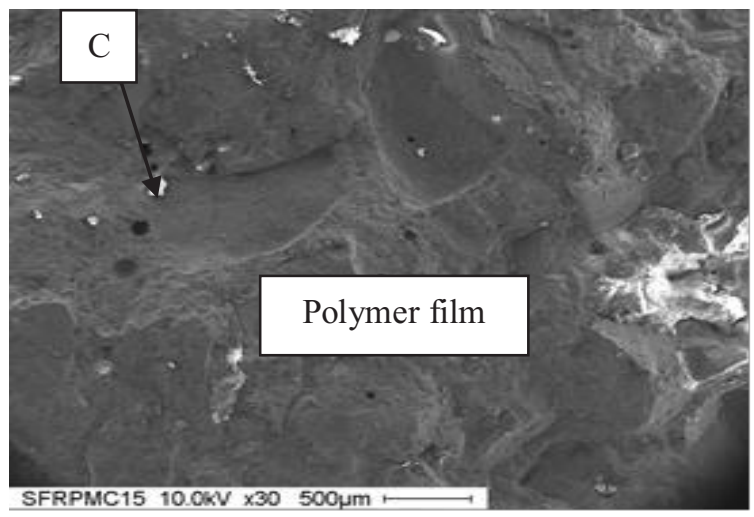

Fig. 2. Microstructure on mix 43 specimen (2.5\% acrylic emulsion polymer, 8.0 silica fume, $1.5 \%$ steel fiber and $0.50 \mathrm{w} / \mathrm{c}$ ratio).

As shown in Fig. 3, the concrete constituents are not compactly joined leads to lower flexural strengths. The acrylic emulsion polymer cement paste (Fig. 3) displays a fishingnet or honeycomb structure. There are ettringite or big pores are present. Furthermore, the polymer films in Fig. 3 are thicker and more coherent than Figure 2. Thus steel fibers can be pulled out more easily in steel fiber reinforced acrylic emulsion polymer modified concrete-21 (mix 63), and flexural strengths then become lower.

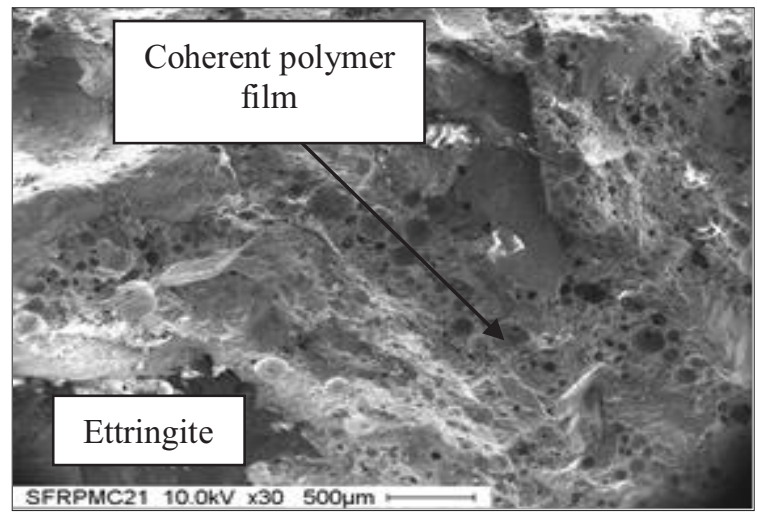

Fig. 3. Microstructure on mix 63 (4.0\% acrylic emulsion polymer polymer, $8 \%$ silica fume, $1.5 \%$ steel fiber and $0.50 \mathrm{w} / \mathrm{c}$ ratio).

Fig. 4 and Fig. 5 show typical SEM microstructures through the combination of several photos, and some inkling of transition zone (ITZ) can be discovered. The results for various ages from SEM are explained as follows [12]. 

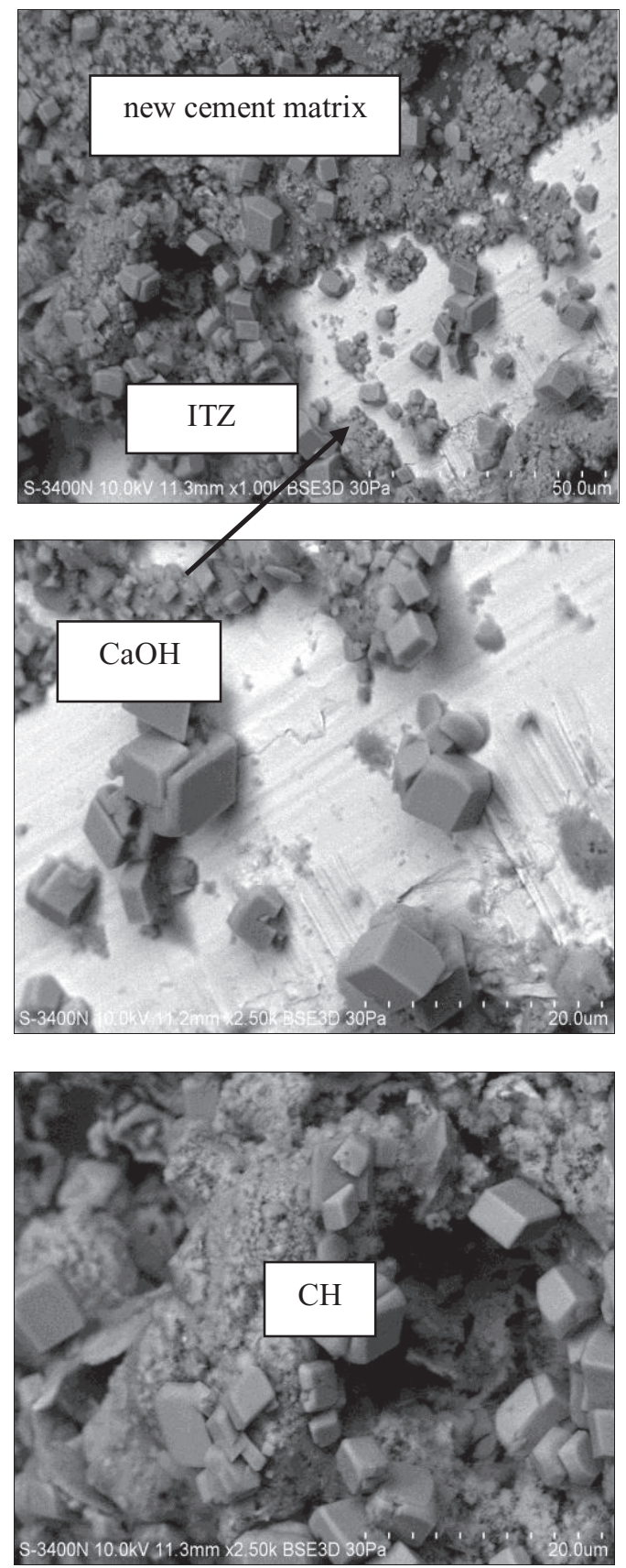

Fig. 4. Spot 1 for mix 43 (optimum) at 1 day curing with three different spots. 

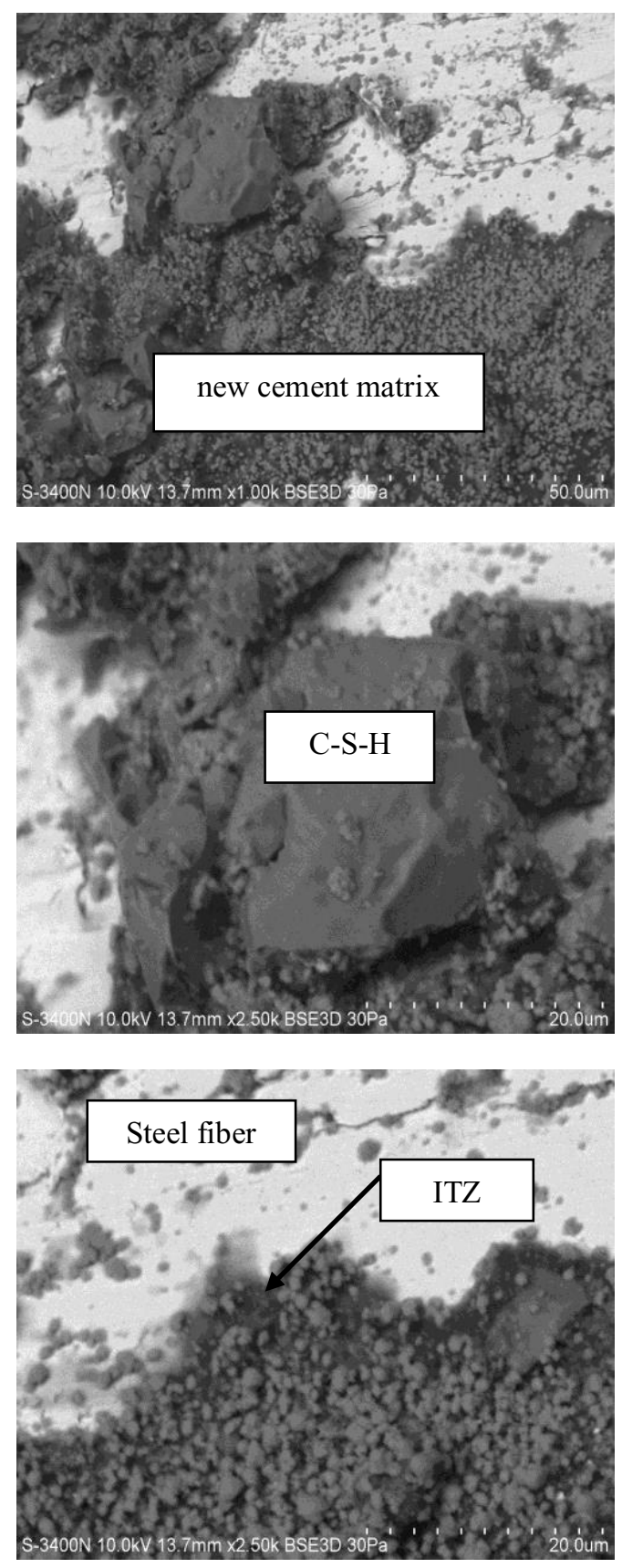

Fig. 5. Spot 1 for mix 43 (optimum) at 7 days curing with three different spots.

Morphology examination by using SEM has been conducted on composite system that had been produced. At the edge of the aggregate, there are much unhydrated cement particles and products of initial hydration. From SEM inspection, a few C-S-H gels can be seen, but the hydration products of $\mathrm{C}-\mathrm{S}-\mathrm{H}$ increases quickly with the increase in curing age. 
At age of 7 days, the unhydrated cement particles and products at the age of 1 day are transformed into C-S-H at the age of 7 days. The needle-shaped C-S-H increased and gradually formed the gel, with needles growing into the pore space just like mesh. At this age, silica fume particles still does not react, but among the hydration products, pore existed.

At the edge of the aggregate, there will still much hydration products caused by the pozzolanic reaction. Due to the mutual stack of these hydration products, it contained plenty of pores. Beyond this range, it can be found that the previous pores are now filled with the hydration products of pozzolanic reaction, making the previous mesh structure turn into plain structure. This phenomenon is more obvious as curing age increased. Moreover, from SEM microstructures, the porosity decreased as the distance from the aggregate edge increased. From the observation silica fume particles kept on breaking and dropping off, and needle-shaped C-S-H is growing in it, in addition of acrylic emulsion polymer into the concrete.

The addition of emulsion polymer causes a plasticizing effect on the cement paste to some extent [13]. Along with cement hydration, the agglomeration of emulsion polymer particles and film formation absorbed on cement occur, which effectively changes the microstructure by compacting the interfacial zones among phases. Finally, a structure of dense interpenetrating network is formed, involving cement hydrates, fine aggregates, and emulsion polymer membranes. From the microstructural analysis of composite, it is observed that using acrylic emulsion polymer modification, through application of high performance $8 \%$ of silica fume, caused a reduction in the porosity of aggregate-matrix ITZ region, compared to unmodified concrete.

\section{Conclusions}

In addition to porosity reduction, it is observed that polymer modified the hydration products in the steel fiber-matrix ITZ. The modification is simpler in concrete with richer cement matrix that is concrete elaborated with silica fume and steel fiber reinforcement improve the bonding strength and this influences directly on mechanical properties of SFRPMC specimens. In addition, the acrylic emulsion polymer can form continuous polymer films inside the concrete, which boosted toughness and compact degree of interface transition zone. The acrylic emulsion polymer could trigger steel fiber and the cement paste to a tight mutual connection. It as well alleviated the macro and micro defect of interface transition zone between fiber and cement paste, strengthening the role of fiber regarding toughness and crack resistance properties. For the SFRC with acrylic emulsion polymer, the impermeability is modified due to the combined effects of plasticizing and filling. At very low $\mathrm{p} / \mathrm{c}(<2.5 \%)$, adding emulsion polymer has little effect on the impermeability. Further addition of emulsion polymer leads to a significant increase in the impermeability. It is believed that the filling effect plays a dominant role in increasing the impermeability when their dosages are high.

This work has been gratefully funded by the University College of Technology Sarawak (UCTS) Grant.

\section{References}

1. ACI Committee 548, Guide for use of polymers in concrete, (ACI International, 2003)

2. S. Popovics, Polymer-modified concrete Detroit, (Concret Institute (ACI), Michigan, 1987) 
3. N. Banthia, Proceedings of FIBCON2012, (ICI, Nagpur, India, 2012)

4. M. Victor, A.E. Flavio, Cement Concrete Res. 42, 1417-1427 (2012)

5. R. Wang, C. Meyer, Cement Construction Composite 34, 975-981 (2012)

6. S. Zhong, H. Yuan, Chemistry Industry Press (2003)

7. Y. Ohama, Proceedings of the Third Asia Symposium on Polymer in Concrete, (Shanghai, 2000)

8. V. Dionys, C. Lech, Cement Construction Composite. 27, 926-33 (2005)

9. X.M. Kong, C.C. Wu, Y.R. Zhang, J. Li, Constr. Build. Mater. 38, 195-203 (2014)

10. M. Aziz, P. Paramasivam, S. Lee, International Journal Cement Composite Lighweight Concrete 3, 123-32 (1981)

11. G. Li, X. Zhao, C. Rong and Z. Wang, Constr. Build. Mater 24 (7), 1201-1206 (2010)

12. Y.L. Kuo, K.C. Ping, N.P. Yaw, C.Y. Chih, Cement and Concrete Research. 34, 977989 (2004)

13. J. Yang, P. Yan, X. Kong, X. Li, Sci. China Ser. E. 53 (5), 1406-1412 (2010)

14. A. Ayob, M.E. Razali, S. Alias, A.G. Ahmad, D.S.H. Ali, MATEC Web Conf. The 9th International Unimas Stem Engineering Conference (ENCON 2016) 87, 01002, (2017) 